\title{
Josef Smolik
}

\section{Die tschechischen Kirchen in der Krise des Jahres 1938 und während der deutschen Okkupation}

In den Jahren 1938 bis 1945 spielte sich das Leben der tschechischen Kirchen in drei politischen Staatsformen ab: bis München (Ende September 1938) in der Tschechoslowakischen Republik, in der Ersten Republik mit dem Präsidenten Eduard Beneš, seit München bis zu der Okkupation (15. März 1939) in der föderalen Tschechisch-Slowakischen Republik, in der Zweiten Republik mit dem Präsidenten Emil Hácha, und nach der Okkupation bis zum Ende des Krieges im Protektorat Böhmen und Mähren mit dem Staatspräsidenten Emil Hácha. Die Reichsprotektoren in der Periode des Protektorats waren Konstantin von Neurath, Reinhard Heydrich, Kurt Daluege und Wilhelm Frick. Dem Leben der tschechischen Kirchen aus dieser Zeit wurde bisher keine monographische Studie gewidmet ${ }^{1}$.

\section{Die tschechischen Kirchen vor dem Zweiten Weltkrieg}

Für das Verständnis der inneren Situation der tschechischen Kirchen in der Periode 1938-1945 ist eine kurze Übersicht über die geschichtliche Lage in der Ersten Republik nötig. Nach dem Fall der österreichisch-ungarischen Monarchie im Jahre 1918 wurde die römisch-katholische Kirche in der Tschechoslowakischen

1 Wir beschränken uns auf die tschechischen Kirchen. Für die Situation der Kirchen in der Slowakei, die völlig verschieden war, gibt es eine Quellenausgabe: Vatikán a Slovenská republika 1939-1945 (Vatikan und die Slowakische Republik 1939-1945) (Bratislava 1992). In den neuesten tschechischen Publikationen über diese Periode wie T. Pasák, JUDr Emil Hácha (1938-1945) (Praha 1997); Pod ochranou řĩse (Unter dem Schutz des Reiches) (Praha 1998); Cesky fašismus (1933-1939) a kolaborace (1940-1945) (Der tschechische Faschismus und die Kollaboration) (Praha 1999); Václav Černý, Paměti 1938-1945 (Memoiren) (Praha 1992) wird den tschechischen Kirchen keine thematische Aufmerksamkeit gewidmet. Die Forschung war auf diesem Gebiet in der Zeit des Kommunismus völlig einseitig und, was die Kirchen betrifft, ideologisch gefärbt. 
Republik, die die Monarchie bis zum Ende des Krieges unterstützt hat, schwer erschüttert. Die Verluste unter dem Einfluß der „Los von Rom“-Bewegung waren groß. Im Jahre 1920 entstand eine neue Kirche, die Tschechoslowakische Kirche mit 700000 Mitgliedern. Trotzdem blieb die katholische Kirche die Mehrheitskirche mit sechs Diözesen; die wichtigsten waren die Erzbistümer in Prag und Olmütz. Auch die Zahl der Mitglieder der evangelischen Kirchen wuchs. Die größte Kirche unter den Protestanten war die Evangelische Kirche der Böhmischen Brüder mit mehr als 200000 Mitgliedern; die anderen evangelischen Kirchen (Baptisten, Herrnhuter, Kongregationalisten, Methodisten) erreichten kaum 10000 Mitglieder. Auf dem Gebiet der Republik lebte auch die Orthodoxe Kirche, die in Mähren einige Gemeinden hatte.

Die schon erwähnte enge Verbindung der katholischen Kirche mit der Monarchie führte dazu, daß sie sich der tschechischen Gesellschaft entfremdet hat. Die geschichtliche Entwicklung brachte die evangelische Minderheit in den Vordergrund, die sich auf die böhmische Reformation (J. Hus, J. A. Comenius) als Höhepunkt der tschechischen Geschichte und Quelle der demokratischen Ideale der Humanität (T. G. Masaryk) berief. Allmählich gewann jedoch auch die katholische Kirche, besonders in der Zeit der wirtschaftlichen Krise, im öffentlichen Leben einen wachsenden Einfluß. (Sichtbar am Katholikentag 1935, an dem der päpstliche Legat Kardinal Verdier aus Paris teilnahm.) Die Volkspartei, die die Interessen der katholischen Kirche vertrat, war unter der Leitung von Msgr. Jan Šrámek ein festes Mitglied der Regierungskoalition. Mit der kommenden Krise der Jahre 1938-1939 wuchs die Zahl der Mitglieder dieser Partei, die in Prag die viertgrößte war. Die Volkspartei in Böhmen und Mähren unterstützte im Unterschied zu der Volkspartei in der Slowakei (A. Hlinka) das humanitäre Programm von Masaryk und Beneš. Msgr. J. Šrámek war während des Krieges Vorsitzender der Exilregierung in London.

Die Protestanten hatten ihre ökumenische Organisation „Konstanzer Union“, die schon in Österreich-Ungarn aus der Bewegung für die Verteidigung der tschechischen Reformationstradition entstanden war. Daneben entfaltete die im Jahre 1927 gegründete Abteilung der internationalen YMCA ihre Tätigkeit, die sog. Akademische YMCA. Sie versammelte die evangelische Intelligenz, vertrat kritisch die Politik Masaryks und versuchte den Einfluß der protestantischen Tradition in der Öffentlichkeit zu stärken (Emanuel Rádl und Josef Lukl Hromádka).

Nach dem Jahre 1937 wuchs in der Tschechoslowakei das Gefühl der Bedrohung. Die deutsche Minderheit unter der Leitung von Konrad Henlein, die aus dem Reich unterstützt wurde, stellte größere Forderungen. Die Protestanten verfolgten aufmerksam die Theologie und die Entwicklung in den Kirchen in Deutschland (die Theologie der Deutschen Christen, der Prozeß Niemöllers), wie aus den Zeitschriften dieser Zeit ersichtlich ist. Das alles trug zum Gefühl der Bedrohung bei. Die Lage polarisierte sich.

Die tschechischen Protestanten identifizierten sich noch stärker mit der demokratischen Republik, in der sie einen Beweis für die Vorsehung und die Gerechtigkeit Gottes nach dem Fall Österreich-Ungarns sahen. Dies fand Ausdruck in der 
Teilnahme vieler Protestanten am Widerstand während der Zweiten Republik und der Okkupation. In den dreißiger Jahren kritisierte Hromádka die Ablehnung des Offenbarungscharakters des Christentums bei Masaryk, lehnte seine Führungsrolle in religiösen Fragen ab und warnte vor der mythischen Identifizierung der humanitären Demokratie mit dem Reich Gottes. In der Zeit vor München stellten sich jedoch die Protestanten zusammen mit der fortschrittlichen Kulturfront mit ganzem Herzen hinter die Demokratie Masaryks. Wenn es nötig sein sollte, waren sie bereit, die Republik mit Waffen zu verteidigen, wozu ihnen Karl Barth in dem berühmten Brief an J. L. Hromádka eine theologische Legitimation gab².

In der Rundfunksendung am 25. September 1939 faßte J. L. Hromádka die Einstellung der Protestanten, die sich mit jener der Bevölkerung deckte, auf folgende Weise zusammen:

„Mag der Friede oder der Krieg kommen, für uns können es herrliche Tage sein, wenn wir im Glauben an die providentielle Gottesherrschaft über die Völker und Staaten unsere Fehler und Sünden erkennen und die Flagge der Wahrheit und der Humanität, der Gerechtigkeit und der Freiheit hoch emporheben. Laßt uns arbeiten - und wenn es nötig sein wird, laßt uns kämpfen. Laßt uns nicht zittern, empfehlen wir unseren Streit dem Herrn über die Welt und über die Völker. "3

Im Rahmen des Katholizismus, der sich gegenüber der Ersten Republik loyal verhielt, kam es zur Differenzierung verschiedener Standpunkte. Der Prager Erzbischof, Kardinal Karel Kašpar, bemühte sich um die Erhaltung des Friedens und verhandelte mit Lord Runciman, den die britische Regierung in die Republik geschickt hatte. Es tauchten allerdings auch solche Stimmen auf, die in der kritischen Situation der Republik eine Bestätigung ihrer früheren Bedenken sahen, daß der im Jahre 1918 angetretene Weg, der die katholische Mehrheit des Volkes an den Rand abzuschieben versuchte, nicht richtig war. Diese Ansichten fanden bei den katholischen jüngeren Literaten und Intellektuellen Resonan $z^{4}$. Diese Stimmen neigten zu der autoritären Auffassung des Ständestaates. Sie blieben auch in den folgenden Jahren lebendig und fanden Unterstützung durch die Behörden des Protektorats. Trotzdem bestimmten sie in keiner Weise die Haltung der katholischen Kirche in ihren leitenden Kreisen oder der Kirchenmitglieder in den Gemeinden.

\section{Nach München}

Der Präsident E. Beneš trat am 5. Oktober 1939 zurück. An seine Stelle wurde Emil Hácha gewählt. Schon die Wahl zeigt, wie sich die Lage in der kirchlichen Szene geändert hatte. Die katholische Tradition des Landes, die nach 1918 im

2 Barths Brief wurde in Prag am 25. 9. 1938 in der Prager Presse veröffentlicht.

3 J. L. Hromádka, Lidé a programy (Leute und Programme) (Praha 1939) 64.

4 Man kann solche Sätze lesen wie "wir bejahen alles, was Hitler gegen die Kommunisten unternommen hat". Aus dem Werk des Schriftstellers Jaroslav Durych zitiert bei T. Pasák, Český fašismus 177. 
Schatten der hussitischen Vergangenheit stand, trat in den Vordergrund. Dies wurde am zweiten Tag nach der Wahl ganz massiv dokumentiert: In der Prager Kathedrale fand ein feierliches Te Deum statt, bei dem der Prager Erzbischof Kardinal K. Kašpar den Brief des Papstes Pius XI. vorlas, in dem der Papst dem neuen Präsidenten den apostolischen Segen erteilte. Nach dem Ende der Messe verbeugte sich Hácha vor den Reliquien der böhmischen Heiligen, und als Beweis seiner tiefen Hochachtung küßte er den Schleier auf dem Schädel des Heiligen Wenzeslaus.

Hácha war ein katholischer Gläubiger, war katholisch erzogen und unterhielt enge Kontakte mit der katholischen Hierarchie und dem tschechischen katholischen Adel. Dieser katholische Hintergrund hat jedoch in keinem Fall seine tiefe Loyalität zu den demokratischen Traditionen, die T. G. Masaryk und E. Beněs repräsentierten, geschwächt. Hácha war dieser Tradition völlig ergeben, was auch sein Besuch nach der Wahl am Grabe Masaryks manifestierte. Auch Präsident Beneš, der schon im Exil war, grüßte Hácha nach seiner Wahl mit einem Brief. Ebenso fanden die Protestanten in ihrer Wochenzeitung für Hácha ein positives Wort ${ }^{5}$, obwohl einige Ereignisse (die Überführung des Palladiums des böhmischen Landes von Prag nach Jungbunzlau, die Einführung des Kreuzes in den Schulen, die Bemühungen um die Erneuerung der Mariensäule auf dem Alten Ring in Prag) zu der Befürchtung führten, die Gedanken der Gegenreformation könnten wieder lebendig werden. In dieser Situation waren die Protestanten bereit, eine Hilfe in dem Bündnis mit der fortschrittlichen kulturellen Front zu suchen. Diese Befürchtungen, die manchmal in den Zeitschriften einen polemischen Ton auf beiden Seiten hervorriefen, erwiesen sich bald als gegenstandslos. Weder Hácha noch die politische Konstellation im Lande erlaubten es den ausgesprochen extremistischen, eher politischen als kirchlichen Gruppierungen, das Verhältnis zwischen den christlichen Kirchen zu stören. Das sollte sich nach der Okkupation zeigen.

\section{Die Okkupation}

Die Zweite Tschechisch-Slowakische Republik war ein Provisorium, das mit der Okkupation und Errichtung des Protektorats Böhmen und Mähren endete. Die extrem rechtsorientierten Gruppen, die gleich nach der Okkupation den „Nationalen Ausschuß des Heiligen Wenzeslaus" bildeten, um an die Macht zu kommen, hatten ihr Ziel nicht erreicht. Dem Präsidenten Hácha war es gelungen, durch die Bildung der „Nationalen Gemeinschaft“, die die Repräsentanten der politischen Parteien zusammenbrachte (auch die Volkspartei war durch Adolf Procházka vertreten) und die demokratische Tradition fortzusetzen versuchte, die faschistischen Gruppierungen aus der Leitung der Republik auszuschließen. T. Pasák stellte fest,

5 E. Lány, Praesident Hácha, in: Kostnické Jiskry, Jg. XX, Nr. 49 vom 1. 12. 1938, 300. 
daß „schon im Jahre 1939 sich in den böhmischen Ländern eine starke oppositionelle Widerstandsströmung bildete, deren bedeutender Bestandteil die römischkatholische Kirche war, ebenso wie die protestantischen Kirchen "6. Im Jahre 1939 kam es nach der Okkupation von Böhmen und Mähren zu einer Reihe von Massenversammlungen, an denen sich die Vertreter der "Nationalen Gemeinschaft“ und der Kirchen offiziell beteiligten. Am 6. Mai wurden die Reliquien des nationalen Dichters K. H. Mácha aus dem Pantheon im Nationalmuseum auf den Friedhof in Vyšehrad überführt. Der Überführung wohnten in den Straßen Prags 200000 Menschen bei. Das Abschiedswort für den Dichter sprach der Kanonikus von Vyšehrad, Msgr. Bohumil Stašek, der wegen seiner Tätigkeit sechs Jahre im Konzentrationslager verbrachte. An der kirchlichen Versammlung in Theresienstadt am 30. April nahmen 90000 Menschen teil. Der Vorstand der "Nationalen Gemeinschaft" beteiligte sich an der Versammlung zu Ehren von J. Hus auf dem Alten Ring in Prag, die von den evangelischen Kirchen veranstaltet wurde. Die stärkste Protestaktion, an der sich die politische Leitung des Protektorats beteiligte, war die römisch-katholische Wallfahrt bei Taussig am 13. August, zu der sich 100000 Menschen versammelten. Msgr. B. Stašek hielt die Predigt, die die Gestapo als „aufwühlende nationale Rede“ bezeichnete”.

Neben diesen öffentlichen Protesten gab es den illegalen Widerstandskampf, der den Deutschen lange verborgen blieb. E. Beneš und die Exilregierung in London knüpften mit dem Vorsitzenden der Regierung in Prag, dem General Alois Eliáš, und mit dem Präsidenten Hácha Kontakte, wobei die katholische Hierarchie und einige katholische Mitglieder des Adels mithalfen ${ }^{8}$.

Von den Kontakten mit den katholischen Kreisen zeugt auch die Tatsache, daß Präsident Beneš die politischen Repräsentanten der Volkspartei, Msgr. Jan Šrámek und Msgr. František Hála, nach London berief. Sie überschritten illegal in einem Eisenbahnwagen unter einem Kohlehaufen die Grenze bei Ostrau.

Dieser Widerstand, der erst nach dem Angriff auf den Protektor R. Heydrich der Gestapo in vollem Ausmaß bekannt wurde, schockierte die Nationalsozialisten, die mit Gegenmaßnahmen, Verhaftungen, Drohungen und Repressalien, die auch die Kirchen trafen, antworteten ${ }^{9}$. Die Kirchen beteiligten sich an den Protesten gegen diese Maßnahmen und an der Unterstützung der Familien der Verfolgten. Am 5. Juni 1939 wandte sich der Erzbischof von Prag K. Kašpar an den Staatspräsidenten E. Hácha mit dem Gesuch, bei dem Reichsprotektor K. von Neurath für die verhafteten Priester zu intervenieren. In seinem Brief erwähnt er

6 Pasák, Fašismus 256.

7 Pasák, Hácha 76.

8 Aus dem tschechischen Adel beteiligten sich an dieser illegalen Tätigkeit František Schwarzenberg und besonders Zdeněk Bořek Dohalský, dessen Bruder Msgr. Antonín Dohalský Sekretär und enger Mitarbeiter des Prager Erzbischofs K. Kašpar war. Beide Brüder Dohalský, Zdeněk und Antonín, starben in Konzentrationslagern.

9 T. Pasák, Persekuce katolické církve v Praze v letech 1939-1945 (Die Verfolgung der katholischen Kirche in Prag in den Jahren 1939-1945), in: Milenium břevnovského kláštera (Praha 1995) $293 \mathrm{ff}$. 
die brutale Behandlung der Priester. Die Zeit der Okkupation charakterisiert der Erzbischof auf folgende Weise: „Die Verhältnisse sind wahrhaftig sehr traurig, wenn es überhaupt kein Recht gibt." 10 Neben den Protesten und Interventionen für die Verhafteten wurde auch die soziale Hilfe für ihre Familien organisiert, wobei die katholische Caritas eine sehr aktive Rolle spielte ${ }^{11}$.

Neben den Kontakten der katholischen Kreise mit London existierten Kontakte mit der Widerstandsbewegung. Hieran beteiligten sich die Mitglieder der evangelischen Kirchen, besonders der Kirche der Böhmischen Brüder und der Herrnhuter. Viele Widerstandskämpfer kamen aus der Akademischen YMCA. Ihre Sekretäre (Jaroslav Šimsa, Rudolf Mareš, Miloslav Kohák, Jaroslav Valenta) waren von Anfang an im Dienste des Widerstandes und brachten in diesem Kampf das größte Opfer, ihr Leben. Auch viele evangelische Familien (besonders in Nordostböhmen) bildeten ein Netz der Widerstandsbewegung.

\section{Die Motivation der Widerstandstätigkeit der Christen}

Die Frage nach der Motivation der Kirchen und der Christen für die Teilnahme an dem Widerstand entfachte nach dem Zweiten Weltkrieg eine rege Diskussion. Der evangelische Pfarrer B. Pospîsil schrieb, daß „die Einstellung der Kirchen im Kampf gegen den Faschismus nicht nur aus Solidarität geschah. Vielmehr handelte es sich um die Identifikation mit dem Volk, wobei die Kirche fast ihre christliche Identität verloren hat [...] Die einfachen Mitglieder und die Leiter der Kirche kämpften zwar tapfer ihren Kampf, aber sie waren nicht fähig, ihre Aufgabe scharf genug in der Perspektive ihres Glaubens zu sehen und ihr Handeln theologisch zu motivieren"12. Es gibt keinen Zweifel daran, daß die Tyrannei des Nationalsozialismus den tschechischen Nationalismus erregte und förderte, der dann in das politische Leben eingedrungen ist, wie sich das nach dem Krieg bei allen Parteien gezeigt hat. Die Kirchen sind allerdings diesem blinden und fanatischen Nationalismus nicht verfallen, was unter anderem die Stimmen gegen die Entfernung der Sudetendeutschen aus den kirchlichen Kreisen beweisen.

Bei den Mitgliedern der Evangelischen Kirche, die sich in der Akademischen YMCA versammelten, war das Verständnis des Evangeliums eng mit den Dimensionen der Menschlichkeit, der Menschenwürde im universal menschlichen Hori-

10 Zitiert bei Pasák, Hácha 105.

11 Diese Arbeit organisierte der Feldkurat der tschechischen Armee, Oberst Jaroslav Janák. Hácha widmete diesem Zweck 500000 Kronen. Dieses Geld wurde auf Grund der Instruktion des Premiers A. Eliáš in der Landesbank auf die Namen „Heiliger Wenzeslaus, Heilige Ludmila, Heiliger Prokop" deponiert.

$12 B$. Pospišill, Službou k svobodě (Durch den Dienst zur Freiheit), in: Od reformacek zítřku (Von der Reformation zum Morgen), hrsg. v. A. Molnár, J. B. Souček u. a. (Praha 1956) 179212, hier 195. 
zont verbunden. Der Nachlaß von Jan Šimsa erlaubt einen Einblick in die Tiefen des biblischen Glaubens, der Menschlichkeit und der Versöhnung, die die nationalen Grenzen überwinden ${ }^{13}$. Bei der katholischen Hierarchie und dem Adel wirkten sicher die Befürchtungen um ihre Stellung mit, aber dies war meiner Meinung nach nicht die eigentliche Motivation ihres Handelns. Im Katholizismus blieb die Idee der böhmischen Staatlichkeit lebendig, wie sie die Tradition des Heiligen Wenzeslaus in der tschechischen Interpretation darstellte. Die böhmische Staatlichkeit existierte lange vor der Ersten Republik Masaryks und auch vor Österreich-Ungarn. Die Verteidigung dieser Kontinuität bedeutete zugleich ein Bekenntnis zu dem christlichen Universalismus, der die nationalsozialistische Ideologie verneinte.

\section{Die Ära Heydrich}

Der Druck auf das öffentliche Leben wurde unerträglich, als im Jahre 1941 R. Heydrich den Reichsprotektor von Neurath ersetzte. Der Antritt Heydrichs bedeutete den Fall des Generals A. Eliáš, die Unterbrechung der Kontakte mit London und eine tiefe Krise zwischen Beneš und Hácha. Beneš forderte nach dem Fall von Eliáš den Rücktritt Háchas. Hácha blieb in seinem Amt, um die Verfolgung zu mildern. Alles gipfelte in dem Attentat auf den Reichsprotektor Heydrich. Das Attentat war die vorher in Einzelheiten geplante Tat des breiten Netzes der Widerstandsbewegung, an der sich viele Christen beteiligten. Er wurde auf Befehl von London durchgeführt mit dem Ziel, bei der britischen Regierung die Anerkennung der Tschechoslowakei in ihren Grenzen vor München zu erreichen. Bei der Vorbereitung und - nach dem Erfolg des Attentats - beim Verstecken der Täter spielte die Orthodoxe Kirche eine wichtige Rolle, vertreten durch ihre Geistlichen Vladimír Petřek und Václav Alois Čikl von der Kirche des Cyrill und Methodius, die Leitung der Kirche Bischof Gorazd (Matěj Pavlík) sowie den Laien Sonnevend. Alle wurden zum Tode verurteilt ${ }^{14}$. Bei der Ausrottung des Dorfes Lidice - die deutsche Antwort auf das Attentat - lehnte der römischkatholische Priester das Angebot ab, sein Leben zu retten, und starb mit den Mitgliedern seiner Gemeinde.

$13 \mathrm{~J}$. und $M$. Šimsa, Úzkost a naděje (Praha 1969). Auch Jaroslav Valenta, der im Widerstand die Verbindung mit Beneš und Hácha besorgte, war ein tiefgläubiger evangelischer Christ. 14 J. Šuvarský, Biskup Gorazd (Praha 1992). 


\section{Die Juden und das kirchliche Leben}

Der Amtsantritt Heydrichs bedeutete für die Juden im Protektorat das letzte tragische Kapitel. Als Heydrich nach Prag kam, lebten auf dem Gebiet des Protektorats 88105 Juden. Nach der Konferenz von Wannsee am 29. Januar 1942 wurde Theresienstadt zur Durchgangsstelle in das Konzentrationslager Auschwitz, wo die Juden liquidiert wurden. Von den 86934 Gefangenen in Theresienstadt, die nach Osten deportiert wurden, überlebten nur 3097. Alle antijüdischen Maßnahmen waren Angelegenheit der Deutschen, denn in den Augen des Besatzers „lehnten es die Tschechen ab, sich mit dem jüdischen Problem auseinanderzusetzen Juden sind ihre Freunde"15. Einige Pfarrer versuchten, die Juden durch die Ausstellung von Taufscheinen zu retten, was entdeckt wurde und wofür sie ins Konzentrationslager kamen.

Das Leben der Kirchen lag unter der Kontrolle der Abteilung für Kirchenfragen bei der Gestapo in Prag (Petschek Palais). Die Kirchen lebten „in den dunklen Katakomben des Schweigens, Leidens und der Erniedrigung der Menschlichkeit" ${ }^{16}$. Nach München verloren die Kirchen ihre tschechischen Gemeinden im Sudetengebiet; es blieb nur eine administrative Verbindung. Viele Mitglieder der kirchlichen Hierarchie, Pfarrer und Prediger, kamen in Konzentrationslagern um. Dasselbe gilt auch von den Hunderten von Kirchenmitgliedern, die sich an den Widerstandsbewegungen und an der Unterstützung der Partisanen beteiligten. Die Kirchen stehen vor der Aufgabe, dieses Kapitel ihrer Geschichte zu verarbeiten. Die Zensur beschränkte das öffentliche Reden und die Presse. Die kirchlichen Zeitschriften versuchten, ihre Tradition fortzusetzen, sie nahmen zögernd die Instruktionen der Zensur an. Die Arbeit mit den Kindern und mit der Jugend, die in den selbständigen Vereinen organisiert war, mußte in die kirchlichen Strukturen eingegliedert werden, YMCA und alle Vereine wurden aufgelöst. Die theologischen Fakultäten waren wie alle Universitätsfakultäten den ganzen Krieg über geschlossen. Die Kirchen organisierten das illegale Studium der Theologie auf ihrem Boden. Die Evangelisation und die Mission konnten sich nicht entwickeln. Die Synoden fanden zwar statt, waren jedoch der Öffentlichkeit nicht zugänglich. Das Leben der Kirche konzentrierte sich auf den Gottesdienst und auf die soziale Arbeit (P. Pitter). Die Teilnahme an dem Leben der Kirche wuchs, auch die finanzielle Opferbereitschaft nahm zu. Unter den Protestanten kam es in dieser Zeit zu einer breiteren Diskussion über die Möglichkeiten der ökumenischen Annäherung und der Einheit der Kirchen, an der sich die Kirche der Böhmischen Brüder, die Methodisten und die Herrnhuter beteiligten.

15 Zitiert nach Pasák, Hácha 182.

16 Die Formulierung des Synodalseniors der Kirche der Böhmischen Brüder aus der Zeit des Protektorats, J. Křenek, in: Pastoralbrief, Kostnické Jiskry, Jg. XXVII, Nr. 14 (Praha 1945) 76. 


\section{Die Kollaboration}

Nach dem Fall der Regierung des Generals A. Eliáš vergrößerte sich die Gefahr der Kollaboration. Sie fand ihre ideologische Grundlage in der „Idee des Heiligen Wenzeslaus", die sie als die Begründung für die Eingliederung Böhmens und Mährens in das Reich benutzte. Diese Idee wurde von einigen Rechtsextremisten im politischen Leben gefördert, auch einige extreme, nicht offizielle katholische Kreise standen ihr nahe. Schon im März 1939 fuhr eine kleine Gruppe nach Rom mit dem Ziel, über den böhmischen Raum zu verhandeln. Sie wurde von der Vorstellung des böhmischen Kurfürstentums im Reich inspiriert. In den Vorstellungen der Historiker "der konservativen katholisch-universalistischen Observanz" war die Idee des mittelalterlichen Ersten Reiches der deutschen Sprache lebendig. Nach diesen Vorstellungen konnte man das nationale tschechische Interesse in das übernationale, christlich-humanistische Reich integrieren. Den Unterschied zwischen dem Römischen Reich Karls des Großen oder Karls IV. und dem Reich Hitlers sahen sie nicht. Im Hintergrund wirkte auch die Abneigung gegen die politische Auffassung von Masaryk mit.

Man muß unterstreichen, daß die Orientierung an der Tradition des Heiligen Wenzeslaus für die katholische Kirche und für den Staatspräsidenten Hácha keineswegs bedeutete, daß Böhmen und Mähren ihre Autonomie verlieren und in das Reich eingegliedert werden sollten ${ }^{17}$.

Die Tradition des Heiligen Wenzeslaus wurde nicht von den kirchlichen, sondern von den politisch kollaborierenden Kreisen mißbraucht. Die führende Rolle spielte dabei der Mitarbeiter des Präsidenten Hácha, Josef Kliment, der von der dauerhaften Eingliederung der Länder des Protektorats in das Reich überzeugt war. Er beeinflußte den Präsidenten Hácha sehr stark, besonders als sich dessen Gesundheitszustand so sehr verschlechterte, daß er nicht mehr fähig war, Widerstand zu leisten und selbständige Entscheidungen zu treffen.

Die deutsche Propaganda nutzte die Tradition des Heiligen Wenzeslaus während der schweren Krise nach dem Attentat auf Heydrich aus, so daß Präsident Beneš sich im Exil genötigt sah, einen Standpunkt dazu einzunehmen. In seiner Botschaft zum Jubiläum der Ersten Republik am 28. Oktober 1941 mit dem Titel "Heute ist nicht die Zeit des Heiligen Wenzeslaus" schrieb er:

„Ganze Prozessionen der verschiedenen Delegationen - der Arbeiter, Bauern, der Intelligenz, der Künstler werden auf der Prager Burg organisiert, und es werden ihnen nach dem Rezept des berühmten Historikers Prof. Pfitzner die nazistischen Stupiditäten von der Poli-

17 Einen Beweis dafür stellt eine Stelle aus den Memoiren von František Schwarzenberg dar. Nach dem Gespräch mit Msgr. O. Stanovsky bereitete Schwarzenberg für den tschechischen Rundfunk eine Rede über den Heiligen Wenzeslaus vor, deren Ausstrahlung die deutsche Zensur nicht erlaubte. Schwarzenberg lehnte die Anschauung ab, daß Wenzeslaus Böhmen in das deutsche Reich eingegliedert hat. „Das römische Reich sollte ein Weltreich sein, das die gleichberechtigten Völker zusammenschließen sollte. “V. Škutina, Česky šlechtic František Schwarzenberg (Praha 1990) 90f. 
tik des Fürsten Wenzeslaus, von dem deutschen Kaiser Karl IV., von der deutschen Reichsstadt Prag, vom deutschen Großraum und Lebensraum, von der Eingliederung des tschechischen Volkes in das Deutsche Reich vorgelegt. "18

Unter denen, die die deutsche Propaganda in die Prozessionen getrieben hat, wurden die Christen und die Kirchen nicht genannt. Die katholische Kirche interpretierte die Tradition des Heiligen Wenzeslaus nicht im Sinne der Nationalsozialisten.

Um die Christen und Kirchen für die Kollaboration zu gewinnen, benutzten die Kollaboranten noch ein anderes Instrument: die Angst vor dem Bolschewismus. Der Minister für Kultur entwickelte die Initiative zur Gründung der „Liga gegen den Bolschewismus“, die ihm die Leute aus dem kulturellen Leben und aus den Kirchen, von welchen er die Unterzeichnung der Erklärung der Liga verlangte, zu kompromittieren und in die Kollaboration hineinzuziehen half. Der Synodalrat der Kirche der Böhmischen Brüder lehnte die Unterstützung der Liga $\mathrm{ab}$, was dazu führte, daß der Synodalsenior Josef Křenek sein Amt niederlegen und Prag verlassen mußte. Dasselbe Schicksal traf auch den Synodalkurator Antonín Boháč.

\section{Das Ende des Protektorats}

Die Frage des Kommunismus und des Verhältnisses zur Sowjetunion bestimmte auch die letzten Monate des Krieges. Bei den Katholiken und den Protestanten wuchs der politische Konservatismus, die Sehnsucht nach der Rückkehr der Demokratie Masaryks. Nach dem Krieg wurde Josef Beran, der im Konzentrationslager gewesen und für Kontakte mit den anderen Kirchen im Interesse des Weltfriedens offen war, zum Erzbischof von Prag gewählt. Politisch konzentrierte sich der Katholizismus auf die Volkspartei, die der Vorsitzende der Exilregierung in London leitete. Diese Partei öffnete sich ökumenisch allen Christen und stellte die Protestanten vor die Frage, ob sie mitmachen sollten. Die Tschechoslowakische Kirche, die im Protektorat ihren Namen ändern mußte und Tschechisch-Mährische Kirche hieß, wechselte schon im Mai 1945 ihre Leitung. Die Namen der neuen Leitung zeigen, daß in der Kirche in den letzten Monaten des Krieges eine starke Tendenz bestand, sich positiv zu den sozialen Veränderungen zu stellen. Der Priester der Orthodoxen Kirche V. Petřek, der in Belgrad studiert hatte, glaubte an die Erneuerungskraft der Orthodoxie in der Sowjetunion. Der Vatikan nahm eine abwartende Stellung ein. Er sandte den chargé d'affaire R. Fordi nach Prag.

Die Situation war offen. Die tschechischen Kirchen haben ihre Glaubwürdigkeit vor dem Volk erwiesen ${ }^{19}$. Keiner Kirche kann man nationale Untreue oder

18 E. Beně̌, Šest let exilu a druhé světové války (Sechs Jahre des Exils und des Zweiten Weltkrieges) (Praha 1946) $143 f$.

19 In den Zeitungen aus der Zeit nach dem Krieg habe ich nur den Namen eines deutschen 
Unzuverlässigkeit vorwerfen. Während in einigen europäischen Ländern die Haltung der katholischen Kirche nicht völlig klar war, gilt das keineswegs von der katholischen Kirche in der Tschechoslowakei. Im Volk entstand eine neue ökumenische Lage des interkonfessionellen Friedens, obwohl im Widerstandskampf die hussitische Tradition überwog. Dieser hoffnungsvollen Entwicklung, die sich gleich nach dem Krieg beobachten ließ, wurde durch die Ereignisse im Jahre 1948 ein Ende gesetzt.

Prälaten gefunden, der wegen Kollaboration vor das Volksgericht gestellt wurde: Franz Bobe, Probst des Malteser Ordens, den die deutsche Regierung nach dem Tode des Erzbischofs K. Kašpar (1941) als Kandidaten für die Stelle des Erzbischofs aufgestellt hatte. 
